\title{
OREVER|
}

\section{Precificação no Agronegócio: Um Mapeamento Sistemático}

\section{Pricing in Agribusiness: A Systematic Mapping}

\section{Giovane Galvão}

giovanegalvao@gmail.com

Graduado em Tecnologia em Análise e Desenvolvimento de Sistemas pela Universidade Tecnológica Federal do Paraná (2014). Mestrando em Computação Aplicada à Agricultura pela Universidade Estadual de Ponta Grossa.

\section{Simone Nasser Matos}

snasser@utfpr.edu.br

Graduada em Bacharel em Processamento de Dados pelo Universidade Estadual de Ponta Grossa (1993). Mestre em Inteligência Computacional pela Universidade Federal do Paraná (2001) e Doutora em Ciências pelo Instituto Tecnológico de Aeronáutica (2008).

\section{Ezequiel Gueiber}

egueiber@gmail.com

Graduado em Bacharel em Informática pela Universidade Estadual de Ponta Grossa (1998). Mestre em Informática pela Universidade Federal do Paraná (2011).

Recebido em: 22/03/2019

Aprovado em: 13/05/2019
Revista do Programa de Pós-Graduação em Extensão Rural (UFV) 


\title{
OREVER
}

\section{RESUMO}

$\mathrm{Na}$ agricultura, a gestão de custo permite ao agricultor precificar seu produto levando-se em consideração fatores naturais, como condição do clima, do solo e localização da propriedade. Este artigo apresenta um mapeamento sistemático sobre a formação de preço na agricultura. Os objetivos do estudo são analisar se existem ferramentas automatizadas de custeio no contexto agrícola, além de verificar quais métodos de precificação e cultura foram aplicados em trabalhos publicados no período de trinta anos. A principal contribuição deste estudo é levantar informações acerca da gestão de custo no agronegócio para implementar um framework nesse contexto. O resultado da revisão mostrou que existem poucos métodos automatizados de gestão de custos na área agronômica. A pecuária é a atividade que mais apresenta métodos de precificação.

Palavras-Chave: Agronegócio; Gestão de Custos; Métodos de Precificação.

\begin{abstract}
In agriculture, cost management allows the farmer to price his product considering natural factors, such as weather, soil and property location. This article presents a systematic mapping on price formation in agriculture. The objectives of the study are analyzing if there are automated costing tools in the agricultural context, as well as to verify which methods of pricing and culture were applied in published works in the period of thirty years. The main contribution of the work is to raise information on the cost management in agribusiness to implement a framework in this context. The result of the review showed that there are few automated methods of cost management in the agronomic area. Livestock farming is the activity that most presents pricing methods.
\end{abstract}

Keywords: Agribusiness; Costs management; Pricing Methods. 


\section{OREVER}

\section{Introdução}

A formação de preço de venda constitui um desafio para empresários, principalmente pelo fato de que o preço exerce influência direta no consumidor final (CREPALDI, 1998). Além dos custos fixos de produção, como depreciações de instalações, implementos, máquinas e animais, outros fatores influenciam na formação do preço de um produto, tais como legislação, impostos, incentivos fiscais, políticas de exportação, aluguéis, mão de obra, seguros, despesas administrativas, armazenamento e transporte, remuneração, entre outros.

Os proprietários e/ou agrônomos de fazendas utilizam o conhecimento sobre custos para avaliar economicamente suas atividades e verificar os fatores de produção, tais como terra, mão de obra e capital, de maneira eficiente, completa e econômica (LOPES et al., 2004 apud LOPES et al., 2015).

As áreas de ineficiência de produção podem ser identificadas para direcionar os esforços de gerenciamento e/ou recursos tecnológicos, a fim de maximizar os lucros ou minimizar os custos (LOPES et al., 2015). Além disso, as informações de território contribuem para a política de crédito fundiário, que serve para auxiliar trabalhadores, com muita ou pouca terra, a expandir sua produção em busca do lucro (MOREIRA et al., 2016).

Os custos dos produtos agrícolas e também de outras atividades do agronegócio, como a pecuária, são influenciados por fatores naturais, que incluem as condições do solo, o clima, a localização da propriedade, a pastagem em época de estiagem, o uso de determinados implementos maquinários, a ração complementar à pastagem, entre outros, e são eles que determinam a qualidade da terra e o rendimento por hectare das culturas. Outra questão relacionada está ligada aos tributos e créditos dos agricultores que participam de associações e cooperativas, como detalhado no trabalho de Gomes e Cezar (2018). Além disso, existem os custos relacionados ao transporte e os de mão de obra para o trabalho mecanizado na produção agrícola (STAŠOVÁ; BAJUS, 2017).

A identificação e medição de custos, despesas e margem de lucro de uma determinada produção resulta na precificação do produto final (SAVIC et al., 2014). Nota-se, aqui, a importância de conseguir medir todas essas variáveis para a formação de preço desse produto.

Os métodos de custeios são utilizados para realizar a precificação de uma determinada produção. Como exemplo, tem-se o método Curva ABC, que é uma técnica de controle e alocação de custos que permite a identificação dos processos e das 


\section{OREVER|}

atividades existentes; a análise e o controle dos custos envolvidos em um processo ou atividade; e a atribuição dos custos aos produtos, tendo como parâmetro a utilização de geradores de custos (SCHIER, 2011).

A escolha do método para o cálculo dos custos depende da natureza da atividade, podendo ser indústria, comércio ou serviços, do uso ou não de tecnologias no ambiente produtivo e, principalmente, do tipo de produção (em massa, contínua, intermitente, etc.). É importante que o gerenciamento possa determinar qual método de cálculo é cabível para uma tarefa de tomada de decisão específica (STAŠOVÁ; BAJUS, 2017).

Este trabalho está organizado em quatro seções. A seção de Metodologia de Pesquisa descreve o funcionamento do mapeamento sistemático e como se deu sua execução. A seção de Resultados e Discussões apresenta os resultados da pesquisa e suas análises e, por fim, na última seção, relata-se as considerações finais do estudo.

\section{Metodologia de Pesquisa}

Kitchenham (2004) diz que um estudo secundário tem como princípio interpretar, identificar e avaliar todos os resultados importantes de um determinado tópico de pesquisa, e é obtido, segundo Mafra e Travassos (2006), através da análise de diversos estudos primários para que seja possível caracterizar um determinado tema de pesquisa.

O mapeamento sistemático (MS) é classificado como um estudo secundário, tendo em vista que é um método projetado para fornecer uma ampla visão de uma determinada área de pesquisa de estudos primários, que permite analisar, quantificar e identificar os resultados (KITCHENHAM; CHARTERS, 2007; ARKSEY; O’MALLEY, 2005). O MS é recomendado quando existe uma carência de estudos primários e/ou o tema da pesquisa é amplo (KITCHENHAM; CHARTERS, 2007).

O mapeamento sistemático aplicado nesta pesquisa baseou-se no método de Petersen et al. (2008), sendo composto por cinco etapas. A primeira delas consiste na definição das questões que devem ser respondidas ao final da pesquisa. A segunda etapa trata da construção das strings de busca e de como será realizada a condução da pesquisa, incluindo nesta fase a definição das bases que serão consultadas. Na terceira etapa, realiza-se a análise dos artigos obtidos. Na quarta, classifica-se os artigos, conforme as categorias escolhidas para o mapeamento, e por fim, a última fase tem a finalidade de realizar a extração dos dados.

A busca foi limitada a trabalhos publicados a partir de 1988. Esse período foi considerado por motivos de escopo de pesquisa, tendo em vista que os primeiros trabalhos 


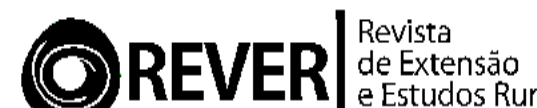

publicados sobre esse assunto ocorreram a partir de 1954, mas não abordavam métodos de precificação no contexto agrícola.

As questões de pesquisa (QP) consideradas nesse mapeamento, foram:

- QP1: Quais são os métodos de custeio aplicados à agricultura?

- $\quad$ QP2: Quais são as culturas em que se aplicam os métodos de custeio?

- $\quad$ QP3: Existem sistemas automatizados para o cálculo de precificação na agricultura?

- QP4: Quais são os tipos de sistemas de precificação na agricultura?

- QP5: Quais os atributos utilizados nos métodos de custeio aplicados na agricultura?

Os repositórios escolhidos para realizar o mapeamento foram: ACM, IEEE Xplore, Portal de Periódicos da Capes, Science Direct e Springer Link. Os mecanismos de busca utilizados nesta pesquisa estão entre os quarenta com melhores avaliações, conforme análise qualitativa realizada por Buchinger, Cavalcanti e Hounsell (2014).

O Quadro 1 apresenta as expressões para a condução desse mapeamento. As strings foram implementadas levando-se em consideração as palavras-chave principais relacionadas ao tema e aquelas recorrentes, conforme leituras em artigos científicos.

Quadro 1: Strings de busca

\begin{tabular}{|c|c|}
\hline String & Expressão \\
\hline 1 & ( ("agriculture" AND "agricultural system") OR "agricultural \\
& $\begin{array}{c}\text { commodity prices" OR "price variability" OR "price level" AND } \\
\text { "agricultural prices") }\end{array}$ \\
\hline 2 & $($ ("cost management" OR "costing methods") AND ("agriculture" OR \\
& $\begin{array}{c}\text { "agricultural system" OR "agricultural commodity prices" OR "price } \\
\text { variability" OR "price level" OR "agricultural prices") })\end{array}$ \\
\hline
\end{tabular}

Fonte: O autor, 2019

As questões de busca foram definidas com o intuito de identificar a existência de ferramentas automatizadas que precificassem produtos agrícolas e as strings geradas, levando-se em consideração as palavras-chave relacionadas ao tema da pesquisa.

Para seleção dos artigos, foram estabelecidos critérios de inclusão (CI), sendo adotados os seguintes: CI1, para Artigos escritos em português ou em inglês; CI2, para 


\section{OREVER}

Artigos completos; CI3, para Artigos publicados a partir de 1988 (30 anos); e CI4, para Periódicos e Conferências com Qualis de estratos superiores (A1, A2 e B1) e inferiores (demais classificações) na área interdisciplinar, ou constantes na lista do Journal Citation Reports (JCR) 2017 ou com indicadores Source Normalized Impact per Paper (SNIP) ou Scimago Journal Rank (SRJ).

Os artigos que atenderam os critérios de inclusão foram novamente avaliados, agora, sob os critérios de exclusão (CE), sendo que os trabalhos que atenderam um ou mais CEs foram retirados do mapeamento. Os critérios de exclusão adotados foram:

- $\quad$ CE1: Artigos duplicados.

- $\quad$ CE2: Artigos que não falam sobre a precificação de culturas na agricultura.

- $\quad$ CE3: Artigos nos quais a expressão de busca seja satisfeita com termos presentes apenas nas referências, biografia do autor, agradecimentos ou conclusão.

Dos 924 artigos encontrados nos repositórios selecionados para o mapeamento, após a análise dos critérios de inclusão e exclusão, restaram 23 trabalhos considerados relevantes para a pesquisa.

Os artigos selecionados nesse mapeamento tiveram seus atributos extraídos conforme o Quadro 2. Tais atributos foram definidos com o objetivo de facilitar a visualização das respostas das questões planejadas no início do mapeamento.

A partir dos 23 artigos selecionados no mapeamento, montou-se uma tabela no software de edição de planilhas (Excel), com colunas referentes aos atributos definidos e linhas contendo as informações extraídas dos trabalhos. Assim, foi possível ter uma visão geral dos resultados da pesquisa. 
Quadro 2: Descrição dos atributos utilizados no mapeamento

\begin{tabular}{|c|c|}
\hline ATRIBUTO & DESCRIÇÄO \\
\hline Autor/Ano & $\begin{array}{c}\text { Nome dos autores do trabalho e o ano em } \\
\text { que foi publicado. }\end{array}$ \\
\hline Finalidade do Trabalho & $\begin{array}{l}\mathrm{O}(\mathrm{s}) \text { objetivo (s) da pesquisa } \\
\text { desenvolvida no artigo. }\end{array}$ \\
\hline Método de Precificação & $\begin{array}{l}\text { Método de custeio inédito desenvolvido } \\
\text { pelos autores. }\end{array}$ \\
\hline Método da Literatura & $\begin{array}{c}\text { O (s) método (s) já existentes na literatura } \\
\text { utilizados no trabalho. }\end{array}$ \\
\hline Cultura & $\begin{array}{l}\text { A cultura agricola em que o método de } \\
\text { custeio foi aplicado. }\end{array}$ \\
\hline Subcultura & $\begin{array}{c}\text { O (s) tipo (s) de cultura utilizado (s) no } \\
\text { trabalho. }\end{array}$ \\
\hline Forma de aplicação do método & Automatizada ou manual. \\
\hline Atributos utilizados no método & $\begin{array}{l}\text { As informações de custo aplicadas no } \\
\text { trabalho destinadas a precificação. }\end{array}$ \\
\hline Trabalhos Futuros & $\begin{array}{c}\text { Melhorias ou novos trabalhos que podem } \\
\text { ser desenvolvidos futuramente. }\end{array}$ \\
\hline Problemas de aplicação do método & $\begin{array}{l}\text { Impedimentos ou limitações do método } \\
\text { aplicado na pesquisa. }\end{array}$ \\
\hline
\end{tabular}

Fonte: O autor, 2019

\section{Resultados e Discussões}

Após a aplicação dos critérios de inclusão, 81 artigos foram selecionados, e em seguida, foram avaliados os critérios de exclusão citados na seção anterior. Para isso, foi efetuada a leitura dos resumos e de algumas seções chaves com o objetivo de categorizar os trabalhos, restando 23 artigos que contemplaram os requisitos estabelecidos.

A Tabela 1 apresenta os resultados do mapeamento. A coluna Estudos Retornados refere-se à quantidade de artigos retornados na condução da busca, a coluna Estudos Potencialmente Relevantes apresenta o número de artigos restantes após a aplicação dos critérios de inclusão e, por fim, a coluna Estudos Selecionados indica quantos trabalhos foram selecionados no mapeamento. 


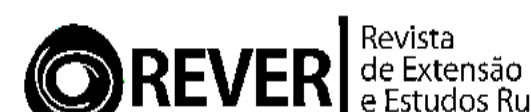 \\ e Estudos Rurais}

Tabela 1: Quantidade de artigos selecionados no mapeamento sistemático

\begin{tabular}{cccc}
\hline Repositório & $\begin{array}{c}\text { Estudos } \\
\text { Retornados }\end{array}$ & $\begin{array}{c}\text { Estudos Potencialmente } \\
\text { Relevantes }\end{array}$ & Estudos Selecionados \\
\hline ACM & 23 & 3 & 1 \\
\hline $\begin{array}{c}\text { IEEE } \\
\text { Xplore }\end{array}$ & 282 & 14 & 6 \\
\hline $\begin{array}{c}\text { Springer } \\
\text { Link }\end{array}$ & 218 & 25 & 5 \\
\hline $\begin{array}{c}\text { Science } \\
\text { Direct }\end{array}$ & 112 & 9 & 10 \\
\hline $\begin{array}{c}\text { Periódicos } \\
\text { da Capes }\end{array}$ & 289 & 30 & 23 \\
\hline Totais & 924 & 81 & \\
\hline
\end{tabular}

Fonte: O autor, 2019

Com os resultados encontrados no mapeamento, é possível realizar uma análise temporal dos anos em que os artigos foram publicados. A Figura 1 compreende essa representação.

Figura 1: Linha do tempo de publicações na área de precificação na agricultura

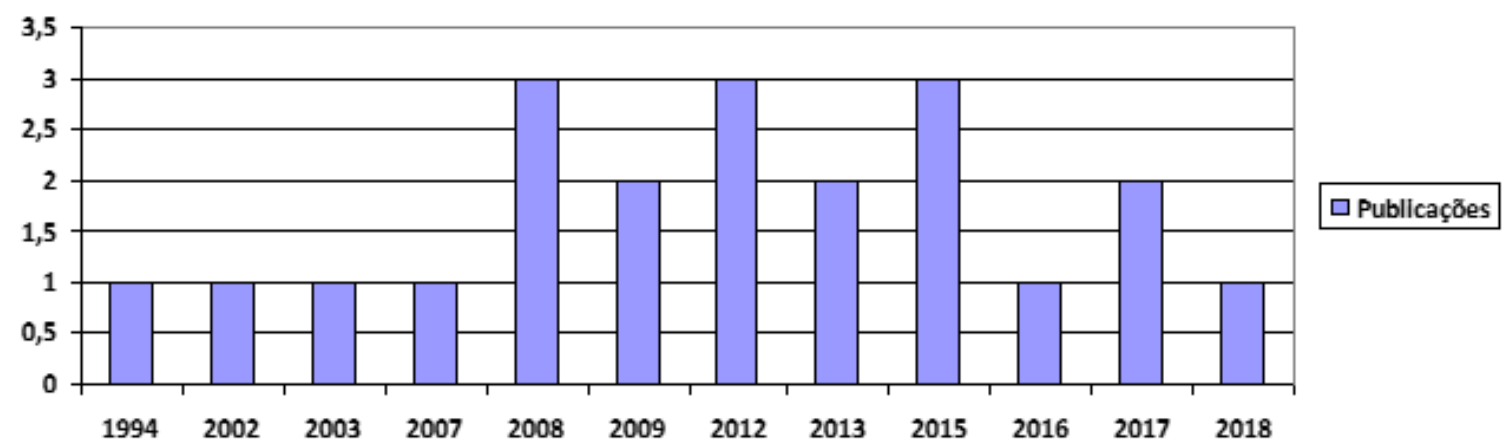

Fonte: O autor, 2019.

A Figura 1 apresenta dois eixos, um deles se refere à quantidade de artigos publicados e o outro corresponde aos anos em que foram encontrados trabalhos referentes ao tema da precificação. Pode-se observar, pelo número de artigos publicados anualmente, que existe uma pequena quantidade de estudos sobre o tema. Além disso, 


\section{OREVER}

constatou-se que a maioria dos métodos de custeio aplicados no contexto agrícola são de execução manual, abrindo, assim, uma lacuna para o desenvolvimento de ferramentas computacionais que precifiquem produtos agrícolas.

Os artigos encontrados no mapeamento e representados em quantidade pela Figura 1 serão apresentados a seguir. Os trabalhos estão relacionados conforme sua proximidade ao tema da pesquisa realizada, ou seja, agrupados de acordo com características em comum.

Dentro do escopo estabelecido nesse mapeamento, a primeira publicação ocorreu em 1994. O trabalho em questão tinha como finalidade gerar previsões de curto prazo acerca dos preços praticados no atacado de Salmão do Japão, desenvolvendo um método de execução manual chamado de Modelo de Resposta ao Impulso (VULKINA; ANDERSON, 1994).

Em 2002, Pataki (2002) desenvolveu um método de precificação manual, baseado em cálculos não convencionais, aplicado à produção de leite, cujo objetivo era construir uma estrutura de controle de custo na produção agrícola primária. Nesse contexto, em 2008, Azevedo e Politti (2008) publicaram uma pesquisa com o intuito de caracterizar o padrão de concorrência no mercado de leite fluido (longa vida e pasteurizado), a partir de evidências sobre os movimentos de preços no varejo e do comportamento das margens de mercado.

Lopes et al. (2015) desenvolveram um modelo chamado de Método Stepwise, baseado em regressão não linear múltipla, que envolve três ou mais variáveis consideradas estimadores, com a finalidade de melhorar a capacidade de predição de determinada informação, para analisar o efeito do nível tecnológico na rentabilidade de 20 unidades demonstrativas (UD) participantes do programa "Balde Cheio", no estado do Rio de Janeiro, no período de janeiro a dezembro de 2011. Nesse estudo, pretendeu-se identificar os componentes que exerceram maior influência sobre os custos total e operacional efetivo e o impacto de cada um deles na receita. Como resultado, concluiuse que o nível tecnológico influenciou na lucratividade e rentabilidade, e os itens componentes do custo operacional efetivo que exerceram maiores representatividades foram alimentação, mão de obra e despesas diversas.

Koekebakker e Lien (2003) adicionaram ao Modelo Jump-Diffusion (BATES, 1991) efeitos sazonais e de maturidade na especificação de volatilidade em culturas anuais, como o trigo. Esse modelo é de execução manual e foi baseado no Modelo de Fackler e Tian (1999). 


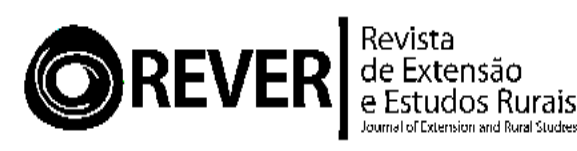

Após quatros anos, Ranogajec, Deže e Karić (2007) desenvolveram um trabalho que tinha como meta a aplicação da Curva $\mathrm{ABC}$ no contexto agrícola. $\mathrm{O}$ desenvolvimento do trabalho se deu na cultura de plantas. González-Gómez e Morini (2009) aplicaram o mesmo método (Curva $\mathrm{ABC}$ ) na mesma cultura, mais especificamente na de plantas ornamentais. Outro trabalho envolvendo Curva ABC foi de Hammer (2016), aplicado na silvicultura.

No ano de 2008, foi implementado um método (manual) chamado de Farm Accountancy Data Network (FADN), o qual descrevia a influência de determinadas categorias de custos na agricultura, como a produtividade e a rentabilidade da produção (SADOWSKI, 2008).

No mesmo ano, Richards et al. (2008) desenvolveram um modelo econométrico baseado no já existente Método de Monte Carlo para a cultura animal de insetos. Outro modelo econométrico foi desenvolvido por Racul e Cimpoie (2012), com o objetivo de precificar o uso de terras, baseado no modelo de regressão não linear e no método estocástico.

Com o propósito de resolver o problema de preços para vários consumidores, projetando modelos de preços combinados e descentralizados com base na teoria da cadeia de abastecimento, a qual estuda o processo de movimentação de bens, do pedido do cliente, passando pela aquisição de matéria-prima, até a produção, Zhang (2009) publicou uma pesquisa que utiliza algoritmos genéticos para tal fim.

No ano de 2012, foram publicados dois trabalhos referentes à temática desse mapeamento. O primeiro, visando à previsão dos preços de vegetais (NASIRA; HEMAGEETHA, 2012), utilizou o Método Backpropagation, e o segundo, buscando prever os preços de alimentos como óleo, carne, ovos, arroz, feijão e açúcar (ZONG;ZHU, 2012), empregou o Método Gray Prediction.

Em 2013, foi publicado um trabalho relacionado à precificação da cultura da banana por Ogbonna et al. (2013). Dois anos após, verifica-se um trabalho relacionado à gestão de custo com pimentões (YE LU et al., 2015) e outro com batatas (TUFA et al., 2015).

O trabalho mais recente encontrado nesse mapeamento foi o de Leshed et al. (2018), que teve como objetivo a criação de uma ferramenta voltada para pequenos cafeicultores de cooperativas com a finalidade de precificar suas produções.

Dentre os problemas da aplicação dos métodos relatados nos artigos selecionados, destacam-se: descontinuidade de preços futuros, tamanho das propriedades, variação de 


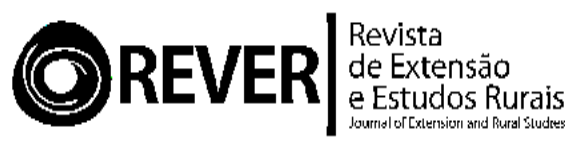

custos médios para segmentos diferentes aos das pesquisas e variações de tributação, considerando determinado contexto agrícola.

Entre os artigos selecionados no mapeamento, poucos sugeriram trabalhos futuros. Racul e Cimpoie (2012) relataram a possibilidade de replicar o estudo para terras com diferentes designações, alterando, assim, as variáveis que geram algum tipo de custo.

Nasira e Hemageetha (2012) citam a importância de melhorar a acurácia do algoritmo desenvolvido no estudo, e Zong e Zhu (2012) falam em estudar a redução do MAPE enquanto os dados não são estáveis.

Zhang e Huang (2013) deixam, como proposta para trabalho futuro, a adição de suposições complexas para a função de custo e de demanda, com o objetivo de resolver o custo-base do modelo de precificação desenvolvido na pesquisa.

Assim sendo, para as questões de pesquisa definidas no início do mapeamento, verificam-se as seguintes respostas:

A. QP1: Quais são os métodos de custeio aplicados à agricultura?

O objetivo dessa questão foi levantar quais os métodos que já foram utilizados no ambiente agronômico para gestão de custos. Pode-se perceber que alguns trabalhos utilizaram métodos da literatura, ou seja, modelos baseados em outros já publicados, como Curva ABC, regressão, Monte Carlo, estocástico, entre outros, e os demais criaram métodos próprios de precificação, o que se chamou de métodos de precificação nesta pesquisa.

Os métodos da literatura frequentes nos artigos selecionados no mapeamento são apresentados na Tabela 2 abaixo.

Entre os 15 métodos encontrados nos trabalhos publicados, percebe-se que o mais utilizado é o de regressão. Vukina e Anderson (1994) utilizam o modelo de regressão para gerar previsões de preços, já Racul e Cimpoie (2012), aplicam a metodologia com o objetivo de descobrir as determinantes de precificação do uso da terra.

Ogbonna et al. (2013) usam o método de regressão para estimar custos na produção de banana; Lopes et al. (2015), através da regressão linear múltipla, conseguem analisar a rentabilidade de fazendas produtoras de leite; e Hammer (2016), Saltone et al. (2016) e Raja et al. (2017) utilizam a regressão para tentar prever o rendimento e o preço dos produtos. Logo, percebe-se que a utilização desse modelo é importante para estimar e gerar previsões de custos de determinado contexto agrícola. 
Tabela 2: Frequência de métodos/modelos encontrados nos artigos

\begin{tabular}{|c|c|c|}
\hline Número & Método/Modelo & Ocorrência \\
\hline 1 & Curva $\mathrm{ABC}$ & 3 \\
\hline 2 & Método Backpropagation & 1 \\
\hline \multirow[t]{2}{*}{3} & Método de Espaço de & 1 \\
\hline & Estado & \\
\hline 4 & Método de Estimativa & 1 \\
\hline 5 & Método de Houck & 1 \\
\hline 6 & Método de Kung-Vaccaro & 1 \\
\hline 7 & Método de Monte Carlo & 1 \\
\hline 8 & Método de Regressão & 7 \\
\hline 9 & Método Estocástico & 2 \\
\hline 10 & Método Estrutural & 1 \\
\hline 11 & Método Gray Prediction & 1 \\
\hline 12 & Modelo Fackler e Tiann & 1 \\
\hline \multirow[t]{2}{*}{13} & Modelo de Previsão PSO- & 1 \\
\hline & BP & \\
\hline 14 & Programação Linear & 1 \\
\hline 15 & Teorema de Kantorovich & 1 \\
\hline
\end{tabular}

Fonte: $\mathrm{O}$ autor

B. QP2: Quais são as culturas em que se aplicam os métodos de custeio?

O objetivo dessa pergunta foi verificar em quais culturas foram aplicados os métodos de custeio elencados no mapeamento. A Figura 2 apresenta o resultado e é possível perceber que a cultura mais recorrente nos trabalhos foi a pecuária.

Figura 2: Culturas encontradas nos artigos selecionados no mapeamento

\section{Cultura X Trabalhos}

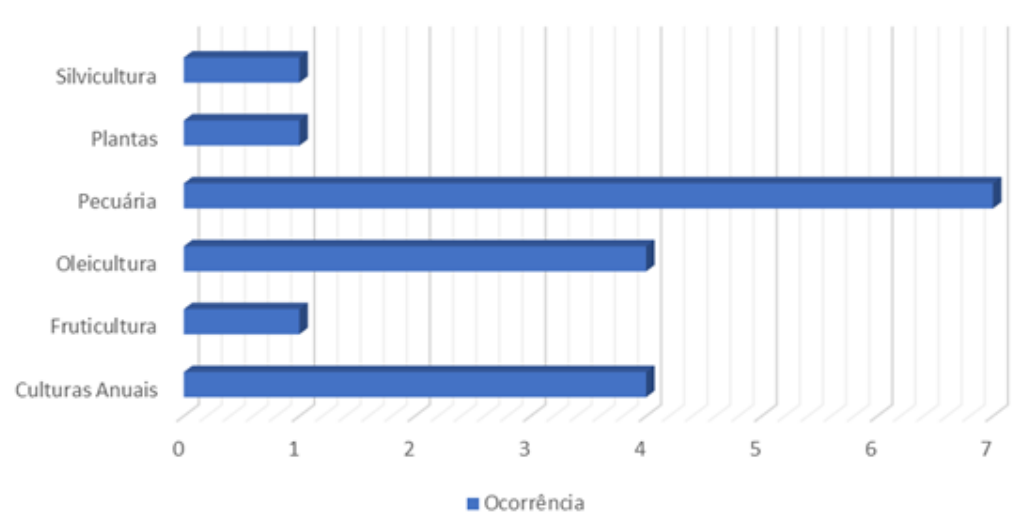

Fonte: O autor, 2019. 


\section{OREVER}

Dentre as quatro culturas anuais que apareceram nos artigos elencados nesse mapeamento, destacam-se o arroz, o café, o feijão e o trigo. No trabalho de Koekebakker e Lien (2003), foi utilizada a cultura anual do trigo para desenvolver um modelo que considera efeitos sazonais e de maturidade na gestão de custo. Com a utilização dessa mesma cultura, Stašová e Bajus (2017) precificaram o custo total de terras agrícolas em euros, além dos ganhos e as perdas por hectare para toda a produção agrícola.

A cultura do café está presente nos estudos de Leshed et al. (2018), em que se observou cafeicultores do Peru participando do desenvolvimento de uma ferramenta baseada em um modelo de custos da produção de café. O intuito era automatizar extensas planilhas geradas pelas cooperativas de café, e a solução contou com a participação de cafeicultores, visando à aplicação de técnicas da Interação Humano-Computador (IHC), como as de acessibilidade.

Em relação à fruticultura, foi encontrado o trabalho de Ogbonna et al. (2013). O estudo considerou os preços dos cachos de banana e as características de cada tipo de banana, por exemplo, a coloração, o tamanho, a maturação, o defeito, a variedade e o comprimento, para estimar os custos de produção dessa cultura.

Quanto à oleicultura, foram levantados os trabalhos de Nasira e Hemageetha (2012), Ye Lu et al. (2015) e Tufa et al. (2015). No primeiro trabalho, foi desenvolvido um modelo de preços para vegetais, em especial, para o tomate, utilizando técnicas de redes neurais. No segundo, aplicou-se o modelo PSO para realizar previsões sobre os preços de pimentões produzidos. E no terceiro, foram formulados métodos de produção de batatas de semente de menor custo para a Etiópia, aplicando-se técnicas de programação linear no desenvolvimento.

A cultura que mais foi abordada nos trabalhos publicados foi a da pecuária, entre eles se encontram os estudos de Vukina e Anderson (1994), com a temática do salmão; Pataky (2002), Azevedo e Politi (2008) e Lopes et al. (2015), sobre a produção de leite; Richards et al. (2008), sobre insetos; e Saitone et al. (2016), sobre bezerros e novilhos.

Com relação à cultura de plantas, Ranogajec, Deže e Karić (2007) e GonzálezGómez e Morini (2009) trabalharam com as ornamentais. Na silvicultura, Hammer (2016) sugeriu uma configuração orçamentária para custos operacionais utilizando o método da Curva ABC em unidades organizacionais estaduais.

C. QP3: Existem sistemas automatizados para o cálculo de precificação na agricultura? 


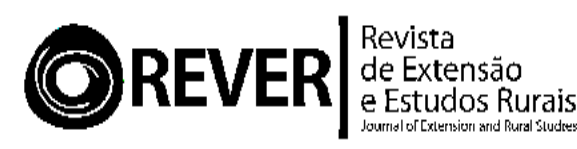

Foi observado que, dos 23 trabalhos selecionados nesta pesquisa, apenas quatro utilizam um modelo de precificação automatizado, logo, a maioria dos métodos utilizados são de natureza manual.

Os modelos automatizados presentes nos trabalhos de Nasira e Hemageetha (2012), Ye Lu et al. (2015), Raja et al. (2017) e Leshed et al. (2018), nas quatro ocorrências, consistem em aplicações específicas e não frameworks. Esses sistemas utilizam atributos relacionados aos custos gerados no processo de produção de uma determinada cultura.

Os autores Nasira e Hemageetha (2012) desenvolveram um sistema de previsão de preços de vegetais, com foco em tomates, utilizando o método de Backpropagation. Além disso, utilizou-se a ferramenta MatLab para a automatização.

Já o trabalho de Ye Lu et al. (2015), teve como finalidade a previsão de preços da produção de pimentões verdes, através do Modelo de Previsão PSO-BP.

Em Raja et al. (2017), utilizando-se a regressão não linear, foi apresentada uma tentativa de prever o rendimento e o preço da safra que um agricultor pode obter de sua terra, analisando padrões em dados passados. Os produtos analisados foram: amendoim, batata, carne, cebola, óleo, tomate e trigo.

Por fim, Leshed et al. (2018) desenvolveram uma ferramenta para cafeicultores calcularem o custo de sua produção, utilizando um modelo de custo de produção subjacente.

D. QP4: Quais são os tipos de sistemas de precificação na agricultura?

$\mathrm{O}$ intuito dessa questão foi verificar quais seriam os tipos de sistemas de precificação automatizados, se esses modelos existissem.

No Quadro 3, são apresentados os resultados obtidos nesse mapeamento, os autores e os anos em que as pesquisas foram publicadas, o método de precificação utilizado e se ele é automatizado ou não. 


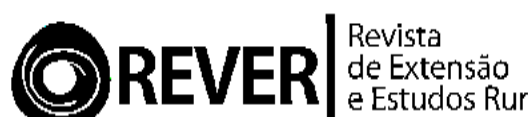

Quadro 3: Sistemas de Precificação na Agricultura

\begin{tabular}{|c|c|c|}
\hline Autor e Ano & Método & Forma de aplicação \\
\hline Vukina e Anderson (1994) & Modelo de resposta ao impulso & Manual \\
\hline Pataky (2002) & $\begin{array}{l}\text { Modelo de cálculo não } \\
\text { convencional }\end{array}$ & Manual \\
\hline Koekebakker e Lien (2003) & Jump-diffusion & Manual \\
\hline Ranogajec, Deže e Karić (2007) & Curva ABC & Manual \\
\hline Sadowski (2008) & $\begin{array}{c}\text { Farm Accountancy Data Network } \\
\text { (FADN) }\end{array}$ & Manual \\
\hline Richards et al. (2008) & Monte Carlo & Manual \\
\hline Azevedo e Politi (2008) & Modelo de Houck & Manual \\
\hline González-Gómez e Morini (2009) & Curva ABC & Manual \\
\hline Zhang et al. (2009) & Algoritmos Genéticos & Manual \\
\hline Racul e Cimpoie (2012) & $\begin{array}{l}\text { Regressão Linear e Método } \\
\text { Estocástico }\end{array}$ & Manual \\
\hline Nasira e Hemageetha (2012) & Método Backpropagation & Automatizado \\
\hline Zong e Zhu (2012) & Método Gray Prediction & Manual \\
\hline Ogbonna et al. (2013) & Regressão & Manual \\
\hline Zhang e Huang (2013) & Teorema de Kantorovich & Manual \\
\hline Lopes et al. (2015) & Método Stepwise & Manual \\
\hline Ye Lu et al. (2015) & Modelo de previsão PSO & Automatizado \\
\hline Tufa et al. (2015) & Programação Linear & Manual \\
\hline Hammer (2016) & Curva $\mathrm{ABC}$ & Manual \\
\hline Saitone et al. (2016) & Regressão & Manual \\
\hline Stasová e Bajus (2017) & -------------------- & Manual \\
\hline Raja et al. (2017) & Regressão não linear & Automatizado \\
\hline Leshed et al. (2018) & $\begin{array}{l}\text { Modelo de custo de produção } \\
\text { subjacente }\end{array}$ & Automatizado \\
\hline
\end{tabular}

Fonte: O autor, 2019.

É possível notar que a maioria dos métodos de precificação empregados na área agronômica são de aplicação manual e baseados em modelos matemáticos de gestão de custos.

Existem poucos trabalhos que desenvolveram modelos de custos na forma automatizada, e os poucos encontrados, como observado no quadro acima, foram descritos na resposta da questão anterior (QP3).

E. QP5: Quais os atributos utilizados nos métodos de custeio aplicados na agricultura?

No geral, observou-se que a maioria dos métodos de precificação levantados nesse mapeamento utilizaram atributos como custos de produção (custos diretos, indiretos, fixos e variáveis), tamanho da área produzida, rendimentos, depreciação, maturação, capital investido, gastos gerados pela mão de obra e impostos.

No Quadro 4, são elencados os atributos utilizados em cada um dos artigos selecionados nesse mapeamento. 
Quadro 4: Atributos dos modelos de precificação

\begin{tabular}{|c|c|}
\hline Autor e Ano & Variáveis de Custo \\
\hline Vukina e Anderson (1994) & $\begin{array}{l}\text { Preço de salmão congelado, salmão de água salgada, Sockeye } \\
\text { (espécie de salmão) congelado e Sockeye salgado. }\end{array}$ \\
\hline Pataky (2002) & $\begin{array}{l}\text { Mão de obra, reparação e manutenção, depreciação, biotecnologia, } \\
\text { atividades veterinárias, inseminação, minerais e alimentação. }\end{array}$ \\
\hline Koekebakker e Lien (2003) & Efeitos de maturação, volatilidade e variações sazonais de preços. \\
\hline Ranogajec, Deže e Karić (2007) & Custos diretos e indiretos por unidade produzida. \\
\hline Sadowski (2008) & $\begin{array}{l}\text { Medida de terras agrícolas, despesas de mão de obra, custos de } \\
\text { consumo intermediários e custos fixos (depreciação). }\end{array}$ \\
\hline Richards et al. (2008) & $\begin{array}{c}\text { Número de aplicações de pesticidas, número de ovos por centímetro } \\
\text { quadrado, insetos adultos por folha e a precipitação. }\end{array}$ \\
\hline Azevedo e Politi (2008) & Custos de produção, coeficiente de tendência e tempo. \\
\hline $\begin{array}{l}\text { González-Gómez e Morini } \\
\text { (2009) }\end{array}$ & $\begin{array}{c}\text { Custos diretos (sementes, estacas, lâmpadas, substratos, potes e auto } \\
\text { abastecimento), indiretos (irrigação, remoção de ervas daninhas, } \\
\text { adubação, enraizamento, corte, estoque e enxerto) e fixos (mão de } \\
\text { obra). }\end{array}$ \\
\hline Zhang et al. (2009) & $\begin{array}{l}\text { Custos com fornecedor, recursos hídricos, custo inicial de } \\
\text { armazenamento, água, varejista e tipos de usuários. }\end{array}$ \\
\hline Racul e Cimpoie (2012) & $\begin{array}{c}\text { Custos de terras agrícolas, variáveis exógenas, matriz de } \\
\text { contiguidade padronizada e coeficiente de autocorreção espacial das } \\
\text { terras. }\end{array}$ \\
\hline Nasira e Hemageetha (2012) & $\begin{array}{l}\text { Preço do tomate de safras anteriores, atuais e previsão de preços } \\
\text { futuros. }\end{array}$ \\
\hline Zong e Zhu (2012) & $\begin{array}{c}\text { Previsão e preços reais de açúcar, carne bovina, carne de porco, } \\
\text { carne de carneiro, ovo. }\end{array}$ \\
\hline Ogbonna et al. (2013) & $\begin{array}{l}\text { Preços dos cachos de bananas, características dos frutos (coloração, } \\
\text { tamanho, defeitos, limpeza, variedade, distância da fonte de fruta, } \\
\text { sazonalidade, comprimento da banana e segurança sanitária) e o } \\
\text { preço implícito dessas características. }\end{array}$ \\
\hline Zhang e Huang (2013) & $\begin{array}{c}\text { Capital fixo investido, horas de trabalho, taxa líquida do capital fixo, } \\
\text { depreciação, salário, custos fixos, imposto de renda, taxa de IVA e } \\
\text { quantidade produzida. }\end{array}$ \\
\hline Lopes et al. (2015) & $\begin{array}{l}\text { Custos fixos (preços de terras, retorno de investimentos, } \\
\text { remuneração de produção e depreciação) e custos variáveis } \\
\text { (alimentação, proteína, energia, fertilização, pesticidas, sal mineral, } \\
\text { mão de obra, reprodução de vacas, maquinário, cuidados com a } \\
\text { saúde do animal, ordenha, custos de familiares dos agricultores). }\end{array}$ \\
\hline Ye Lu et al. (2015) & Preço do varejo de pimentão verde. \\
\hline Tufa et al. (2015) & $\begin{array}{c}\text { Fontes de semente, tamanho, método de armazenamento, método de } \\
\text { brotação, frequência de lavoura, data de plantio e frequência de } \\
\text { capina. }\end{array}$ \\
\hline Hammer (2016) & $\begin{array}{l}\text { Ativos tangíveis de longo prazo, material de escritório, serviços } \\
\text { postais, serviços de telecomunicação, aluguel, compra de outros } \\
\text { serviços, manutenção de bens imóveis e manutenção de veículos. }\end{array}$ \\
\hline Saitone et al. (2016) & $\begin{array}{l}\text { Vacinação de gados, certificação da carne (Angus), gados } \\
\text { desmamados, lotes com alguma variabilidade, lotes com alta } \\
\text { variabilidade, peso e custos fixos de despesas pagas a prazo. }\end{array}$ \\
\hline Stasová e Bajus (2017) & Totais de custos, receita, lucro e prejuízos. \\
\hline Raja et al. (2017) & Tipo de cultura, qualidade (em tonelada), lucros e perdas. \\
\hline Leshed et al. (2018) & $\begin{array}{l}\text { Entrada: Número de hectares por planta de café, método de cultivo, } \\
\text { salário diário dos funcionários, produtividade em kg/ha. Saída: } \\
\text { custos variáveis, custos fixos e custos adicionais. }\end{array}$ \\
\hline
\end{tabular}

Fonte: O autor, 2019 


\section{OREVER/|}

Nota-se que a maioria dos trabalhos utilizam como atributos para seus modelos de precificação os custos envolvendo determinada cultura. As atividades que geram algum tipo de custo, seja de forma direta, indireta ou fixa, são levadas em consideração no cálculo dos modelos. É possível observar essas características nos artigos de Pataky (2002), Ranogajec, Deže e Karić (2007), Azevedo e Politi (2008), Richards et al. (2008), González-Gómez e Morini (2009), Lopes et al. (2015), Tufa et al. (2015), Hammer (2016), Saitone et al. (2016), Raja et al. (2017) e Leshed et al. (2018).

Em alguns trabalhos, observou-se que há comparação de preços, como nos casos das pesquisas de Vukina e Anderson (1994), Nasira e Hemageetha (2012), Zong e Zhu (2012), Ogbonna et al. (2013) e Ye Lu et al. (2015).

Existem também trabalhos relacionados à precificação de terras, como os de Sadowski (2008), Racul e Cimpoie (2012) e Stasová e Bajus (2017), e outros que tratam de características especificas, como maturação, volatilidade e efeitos sazonais (KOEKEBAKKER; LIEN, 2003).

\section{Considerações Finais}

Este artigo apresentou um mapeamento sistemático que permitiu verificar quais são os métodos de custeio já publicados na literatura no decorrer de trinta anos, as culturas agrícolas às quais foram aplicados e sua finalidade.

Primeiramente, foram definidas as questões de busca, as strings e os critérios de inclusão e exclusão de artigos. Dos artigos selecionados, foram extraídos os atributos definidos pela equipe que executou o mapeamento. Foram retornados 924 artigos, dos quais 81 foram considerados potencialmente relevantes, aplicando-se os critérios de inclusão e, após empregar os critérios de exclusão, obteve-se 23 estudos selecionados. Por fim, foi possível responder as questões elaboradas no início do mapeamento.

Este estudo mostrou que existem muitos métodos de execução manual e poucos automatizados, apresentou os atributos utilizados nesses métodos, se houve algum problema na sua aplicação e se existem trabalhos futuros relacionados à temática do trabalho.

Pode-se perceber que o método mais utilizado nos estudos foi o de Regressão e a cultura que apareceu de forma mais frequente nos trabalhos foi a da pecuária.

Com relação aos atributos, nota-se que a maioria dos trabalhos consideram custos fixos, diretos e indiretos nos modelos de precificação, evidenciando que o desenvolvimento de uma ferramenta automatizada na área necessita incluir todas essas 


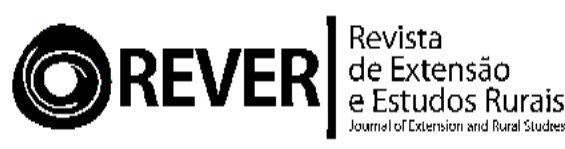

categorias de custo para que se possa realizar a precificação de forma adequada, sendo que ainda há outras pesquisas que fazem a análise dos preços dos produtos já acabados para geração de custos.

Como trabalhos futuros, sugere-se o desenvolvimento de ferramentas automatizadas que auxiliem no custeio de determinadas produções no contexto agrícola, pois, como é possível observar nos resultados deste estudo, a maioria dos modelos são de execução manual. Além disso, sugere-se a implementação dos métodos de custeio Curva ABC, Regressão e Monte Carlo, que são métodos que apareceram nos resultados deste estudo. Ademais, a implementação de um framework torna-se um diferencial nessa área.

\section{Referências bibliográficas}

ARKSEY, H., O'MALLEY, L. Scoping studies: Towards a methodological framework. International Journal of Social Research Methodology: Theory and Practice, 2005.

AZEVEDO, P. F. d., POLITI, R. B. Concorrência e estratégias de precificação no sistema agroindustrial do leite, RESR pp. 767-802, 2008.

BATES, D. S. The crash of '87: Was it expected? the evidence from options markets, Journal of Financial pp. 1009-1044,1991.

CREPALDI, S. A. Contabilidade gerencial, teoria e prática, Atlas, 1998.

EMEROLE, C. O., OSONDU, K. C., ANYIRO, O. C., ORJI, O. A. Trade enhancement characteristics of dessert banana fruits and estimates of transaction costs in okigwe metropolis, imo state nigeria, International Journal of Food and Agricultural Economics (1): 141-150, 2013.

FACKLER, P. L.,TIAN, Y. Volatility models for commodity markets, 1999.

GOMES, E., CEZAR, L. O papel das cooperativas da agricultura familiar no desenvolvimento de políticas públicas. Revista de Extensão e Estudos Rurais, 7(1), 166$186,2018$.

GONZÁLEZ-GÓMEZ, J. I., MORINI, S. A model for cost calculation and management in a multiproduct agricultural framework. the case for ornamental plants, Spanish Journal of Agricultural Research pp. 12-23, 2009.

HAMMER, J. The budgetary process with a use of modern approaches in cost management, ACTA UNIVERSITATIS AGRICULTURAE ET SILVICULTURAE MENDELIANAE BRUNENSIS, 2016.

KITCHENHAM, B. Procedures for performing systematic reviews. Joint Technical Report Software Engineering Group, 2004. 


\section{OREVER|}

KITCHENHAM, B., CHARTERS, S. Guidelines for performing systematic literature reviews in software engineering. EBSE Technical Report, 2007.

LESHED, G., HUANG, M., Mansbach, L. Designing for transparency of cofee production costs, CHI EA '18 Extended Abstracts of the 2018 CHI Conference on Human Factors in Computing Systems, 2016.

LIEN, G., KOEKEBAKKER, S. Volatility and price jumps in agricultural futures prices - evidence from wheat options, American Journal of Agricultural Economics (4): 1018 1031, 2004.

LOPES, M. A., MORAES, F. d., CARVALHO, F. d. M., PERES, A. A. d. C., BRUHN, F. R. P., REIS, E. M. B. The effect of technological levels on profts of milk production systems participating in the "full bucket" program: a multicase study, Semina: Ciências Agrárias (4): 2909-2922, 2015.

NASIRA, G. M., HEMAGEETHA, N. Vegetable price prediction using datamining classi_cation technique, Proceedings of the International Conference on Pattern Recognition, Informatics and Medical Engineering., 2012.

MAFRA, S. N., TRAVASSOS, G. H. Estudos primários e secundários apoiando a busca por evidencia em engenharia de software. 2006.

MOREIRA, D., FERREIRA NETO, J., APARECIDA DE MOURA, R., HELENA TEIXEIRA, T. (2016). Desenvolvimento E Organização Territorial: Uma Análise Das Políticas De Gestão De Terras No Brasil E Na Galícia. Revista de Extensão e Estudos Rurais, 5(1).

PATAKI, J. Methodological aspects of an improvement of calculation information system in agricultural companies, AGRIC. ECON., pp. 130-133, 2012.

PETERSEN, K., MUJTABA, S., MATTSSON, M. Systematic mapping studies in software engineering, EASE'08 Proceedings of the $12^{\text {th }}$ international conference on Evaluation and Assessment in Software Engineering pp. 68-77, 2008.

POPESKO, B., PAPADAKI, S., NOVÁK, P. Cost and reimbursement analysis of selected hospital diagnoses via activity-based costing, EM Economie and Management (3): 50-61, 2015.

RACUL, A., CIMPOIES, D. Land - price determinants using the spatial econometrics modeling in the moldavian real estate market, Management, Economic Engineering in Agriculture and Rural Development (4), 2012.

RAJA, S. K. S., RISHI, R., SUNDARESAN, E., SRIJIT, V. Demand based crop recommender system for farmers, 2017 IEEE International Conference on Technological Innovations in ICT For Agriculture and Rural Development (TIAR 2017).

RANOGAJEC, L., DEŽE, J., KARIC, M. Cost management functioning as a competitiveness of agricultural production, European Association of Agricultural Economists, 2007. 


\section{OREVER|}

RICHARDS, T. J., EAVES, J., MANFREDO, M., NARANJO, S., CHU, C. C., HENNEBERRY, T. J. Spatialtemporal model of insect growth, di_usion and derivative pricing, Agricultural and Applied Economics Association p. 962-978, 2008.

SADOWSKI, A. Economic e_ciency of inputs of production factors in cost management context, Journal of Agribusiness and Rural Development pp. 123- 133, 2008.

SAITONE, T. L., FORERO, L. C., NADER, G. A., FORERO, L. E. Calf and yearling prices

in california and the western united states, CALIFORNIA AGRICULTURE (4), 2016.

SAVIC, B., VASILJEVIC, Z., ĐOR.EVIC, D. Strategic cost management as instrument for improving competitiveness of agribusiness complex, Economics of Agriculture, 2014

STAŠOVÁ, L. H., BAJUS, R. Costs of growing wheat and oilseed rape in slovakia and other v4 countries, Potravinarstvo Slovak Journal of Food Sciences (1): 685-695, 2017.

TUFA, A. H., LOMMEN, W. J. M., TSEGAYE, A., STRUIK, P. C., LANSINK, A. G. J. M. O. Least-cost seed potato production in ethiopia, Potato Research p. 277-300, 2015.

VULKINA, T., ANDERSON, J. L. Price forecasting with state-space models of nonstationary time series: Case of the Japanese salmon market, Computers Math. Applic. (5): 45-62, 1994.

YE, L., LI, Y., LIANG, W., SONG, Q., LIU, Y., QIN, X. Vegetable price prediction based on pso-bp neural network, 2015 8th International Conference on Intelligent Computation Technology and Automation, 2015.

ZHANG, L., WANG, H., HU, Z., YANG, W. A discriminatory pricing model and simulation to different users of eastern route of the south-tonorth water transfers supply chain, 2009.

ZHANG, Y., HUANG, J. H. Cost-based pricing model with value-added tax and corporate income tax for a supply chain network, Applied Mathematical Modelling p. 168-180, 2014.

ZONG, J., ZHU, Q. Z. Apply grey prediction in the agriculture production price, 2012 Fourth International Conference on Multimedia Information Networking and Security. 